\title{
Construcción de la identidad profesional de los formadores de docentes de la Universidad Pedagógica Nacional*
}

\author{
José Pedro Valdez Martínez. \\ (iD http://orcid.org/0000-0002-2673-2846 \\ Universidad Pedagógica Nacional, México
}

DOI: http://dx.doi.org/10.17081/eduhum.19.32.2538

Recibido: 3 de junio de 2016

Aceptado: 30 de agosto de 2016

\begin{abstract}
The construction of the professional identity of teacher trainers from Universidad Pedagogica Nacional

Palabras clave:

Identidad profesional, Formadores de docentes e Investigación.

\section{Resumen}

Este artículo es resultado de una investigación realizada con el objetivo de conocer la construcción de la identidad profesional de formadores de docentes en la Universidad Pedagógica Nacional (UPN), en Tehuacán, Puebla. Se trabajó con metodología cualitativa de corte etnográfico y con categorías etic para recabar información a partir de observaciones, entrevistas y grupos de discusión. En el análisis realizado con triangulación metodológica y de informantes, con apoyo del Software MAXQDA, surgieron categorías emic. Los hallazgos más significativos indican que la identidad profesional está estrechamente vinculada con la docencia como función principal; complementadas por las tareas de asesoría y tutoría, en tanto que la investigación es prácticamente nula. Entre las conclusiones destaca la resiliencia de los formadores como un factor importante en la preparación de los nuevos docentes.
\end{abstract}

Key words:

Professional identity, Teacher trainers and Research.

\begin{abstract}
This paper focuses on research which aims to know the professional identity construction of teacher trainers in Universidad Pedagogica Nacional (UPN) en Tehuacán, Puebla. This research was conducted by applying qualitative methodology, ethnographic in its nature. Interview and discussion groups and categories etic were used in order to collect observation based data. Emic categories emerged during the analysis carried out with methodological triangulation and were based on informers with MAXQDA Software assistance. The most significant results were: professional identity is closely linked to teaching process as the main function and consultancy; and tutoring tasks complementing this research process is almost absent. Teacher trainers resilience stands out as an important factor during new teachers' training.
\end{abstract}

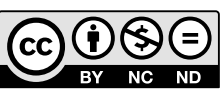

Referencia de este artículo (APA): Valdez, J. (2017). Construcción de la identidad profesional de los formadores de docentes de la Universidad Pedagógica Nacional. En Revista Educación y Humanismo, 19(32), 145-158. http://dx.doi.org/10.17081/ eduhum.19.32.2538

\footnotetext{
* Este artículo forma parte de la tesis "La construcción de la identidad profesional de los formadores de docentes. Un estudio de caso de la Universidad Pedagógica Nacional, Unidad 213, en Tehuacán, Puebla", que se presentó en enero de 2017 para obtener el grado de Doctor en Educación por la Universidad Autónoma de Tlaxcala, México. El Doctorado fue cursado de agosto de 2012 a julio de 2015 con registro como Programa Nacional de Posgrados de Calidad en el Padrón del Consejo Nacional de Ciencia y Tecnología del cual se recibió la beca correspondiente.

1. Doctorado en Educación en la Universidad Autónoma de Tlaxcala, México.jpedrovaldez@hotmail.com
} 


\section{Introducción}

El estudio de las condiciones de los formadores de docentes es relativamente reciente en el ámbito de la investigación educativa y no ha sido tratado con suficiente profundidad en sus diferentes aspectos, como lo demuestran los siguientes estados del arte:

Según Messina (1999) la formación y los saberes pedagógicos de los formadores son ámbitos poco estudiados, en lo que coincide con Vaillant (2002), quien comenta que son escasas las investigaciones y los trabajos que exploran la temática de los formadores. Por su parte, Valdés, Fernández $y$ da Silva (2005) explican que, aun cuando en los últimos años se han incrementado las investigaciones sobre la formación de profesores, los estudios de estados del arte representan aproximadamente el $6 \%$ del total de las investigaciones.

Ahora bien, para abordar el problema de estudio sobre la identidad profesional en este trabajo, se optó por una perspectiva sociológica como fundamento teórico a partir de autores como: Giménez (1997 y 2005); Dubar (2000 y 2002); Cacho (2004); Bolívar, Fernández y Molina (2005); Bolívar (2007), y Mieles-Barrera, Henríquez-Linero y Sánchez-Castellón (2009).

\section{Identidad profesional}

El concepto de identidad resurgió a partir de movimientos sociales que buscaban reconocimiento social y autonomía, como por ejemplo: migrantes, etnias, ancianos, gays, mujeres. Todo esto como una respuesta ante las actitudes de aislamiento y adaptación que diferentes grupos habían asumido ante ellos.

En estas circunstancias, la identidad profesional es considerada como un vínculo o, con otras palabras, es el concepto que los maestros forjan de sí mismos pero en concordancia a su profesión y su trabajo, de manera que sin el marco de las relaciones sociales no es posible esa constitución. Por tanto, la identidad es siempre una correlación con el otro y se define durante la trayectoria profesional, en interacción con los contextos formativos y profesionales por los que cada persona pasa a lo largo de su vida laboral (Gewerc, 2001; Rebollo, González \& García, 2002; García, 2003 y Avalos 2003, Cavada, Pardo \& Sotomayor, 2010).

La construcción de la identidad se logra a partir de un doble proceso: el de atribución, donde las instituciones e individuos están en interacción; y el de incorporación o interiorización activa del individuo mismo, que no puede analizarse sin considerar las trayectorias sociales porque nadie puede construir su identidad al margen de las identificaciones que los otros formulan sobre él (Cattonar, 2001 \& Bolívar et al., 2005). Para su constitución, la identidad requiere, entonces, elementos de la propia historia personal y social, de las instituciones productivas y reproductoras, todo lo cual está ligado a la asimilación de normas, reglas y valores profesionales de acoplamiento entre la elección de lo que se quería ser y lo que en la práctica el oficio es (Gewerc, 2001; Bolívar, 2007). 


\section{Problema de investigación}

En el Acuerdo Nacional para la Modernización de la Educación Básica (Presidencia de la República, 1992), se indica que cada Entidad Federativa debe conformar un sistema estatal de formación para los maestros. También las reformas de principios de esta década que, a través del Acuerdo 592 establece la articulación de la Educación Básica (SEP, 2011) integrando los tres niveles (preescolar, primaria y secundaria) bajo un enfoque por competencias y la reciente Ley General del Servicio Profesional Docente (Cámara de Diputados, 2013) exigen, entre otras cosas, que los docentes de educación básica y media superior logren las competencias indispensables y los perfiles deseables para ejercer la función que les corresponde desempeñar, una vez que han sido contratados por la Secretaría de Educación Pública.

Las tareas que se desprenden de las reformas descritas involucran directamente a las instituciones que forman, capacitan, actualizan y profesionalizan a los docentes de los niveles implicados. Entre estas se encuentra: la UPN y, en específico para este estudio, la Unidad 213 de Tehuacán, Puebla. Por lo cual, se planteó estudiar el problema de la construcción de las identidades profesionales de los formadores de docentes, en las que se contemplan: sus perfiles profesionales, su profesionalización, su aspecto laboral y su vida cotidiana. Tales elementos nos permiten comprenderlos como personas, como trabajadores y como profesionales de la educación.

\section{Método}

En el presente estudio se utilizó la metodo- logía cualitativa por ser la que mejor se asocia con técnicas como la observación, el estudio de casos, la etnografía y la entrevista, las cuales buscan el entendimiento de una realidad mediante un proceso interpretativo (Reese, Kroesen \& Gallimore, 2010). Con este tipo de investigación se trató de dar cuenta de los significados, actividades, acciones e interacciones cotidianas de los sujetos en su contexto.

A su vez, se optó por un enfoque etnográfico para efectuar una descripción de los acontecimientos que tuvieron lugar en la vida individual y del colectivo, a fin de generar interpretaciones y otorgar significados a la cultura de la que forman parte (Reynaga, 2010). Así, se construyó una explicación de la realidad investigada que buscó ser externa e independiente del propio investigador (Hammersley \& Atkinson, 2009).

Los participantes en la investigación sobre la construcción de la identidad profesional fueron los formadores de docentes de la UPN en Tehuacán, Puebla, universidad que fue creada por Decreto Presidencial en 1978 como una Institución de Educación Superior con carácter de Organismo Público Desconcentrado de la SEP y como un sistema de unidades en todo el país. En el Artículo $2^{\circ}$. del decreto citado, se especifica que la UPN tiene por finalidad "prestar, desarrollar y orientar servicios educativos de tipo superior encaminados a la formación de profesionales de la educación de acuerdo a las necesidades del país" (p.98); y en el Artículo $3^{\circ}$ se indica que sus funciones deberán guardar relación permanente 
de armonía y equilibrio, de conformidad con los objetivos y metas de la planeación educativa nacional, enunciando las siguientes: a) Docencia de tipo superior; b) Investigación científica en materia educativa y disciplinas afines; c) Difusión de conocimientos relacionados con la educación y la cultura en general.

Vaillant (2002) considera a los formadores, como profesionales capaces de ejercer la actividad de formación con conocimientos teóricos y prácticos, compromiso e iniciativa para aprender y transformar, que pertenecen a colectivos; los presume, por tanto, como agentes de cambio, mediadores que pueden y deben contribuir a dinamizar la innovación en las instituciones.

De acuerdo con lo anterior, los criterios de inclusión y exclusión que se aplicaron para seleccionar a los sujetos investigados fueron: ser académico de medio tiempo o de tiempo completo y tener un mínimo de tres años de experiencia en la función de formadores de docentes.

Las técnicas utilizadas en este estudio fueron la observación etnográfica, las entrevistas semiestructuradas y los grupos de discusión. En la observación, el etnógrafo siempre fue participante, es decir, formó parte de la escena, ejerciendo influencia, provocando cambios en las actitudes de los docentes y evaluando el material descubierto y presentado. Las observaciones se llevaron a cabo en las aulas durante las horas de clase, así como en otras actividades institucionales tales como reuniones y eventos de trabajo que tuvieron los formadores durante el periodo de observación. Con apoyo de una grabadora y de un cuaderno de notas, se registró la mayor información posible.

La entrevista se consideró complementaria a la observación, pero adquirió un valor insustituible debido a que ambas constituyen un ejercicio básico para la producción de información. También se justifica la complementariedad de dichos métodos con base en los argumentos de control, fiabilidad y significatividad de los datos, porque la observación proporcionó el contraste necesario de la realidad subjetiva comunicada a través de la entrevista; por otro lado, esta última confirió sentido a las acciones a veces incomprensibles que se observaron, o corrigió las inferencias, en ocasiones precipitadas, que se obtuvieron en la observación (Velasco \& Díaz de Rada, 2009; Díaz de Rada, 2011).

Cada entrevista se realizó en consenso con los formadores, así como en los lugares, fechas $\mathrm{y}$ horarios que permitieron el buen desarrollo de la sesión y las mejores condiciones para recabar datos, en un tiempo aproximado de 60 a 90 minutos. Al iniciarla se contaba ya con los datos de la hoja de portada, es decir, con la información general previa a fin de lograr un discurso fluido e inteligente.

Por otra parte, el grupo de discusión se entendió en sí mismo como un método de 
investigación social que se trabajó mediante el diálogo, con la intención de potenciar la interacción acerca del tema objeto de esta investigación en un tiempo determinado. Esto con el propósito de captar la forma de pensar, sentir y vivir de los individuos que conforman al grupo de profesores y de alumnos jefes de grupo. De este modo, el papel del entrevistador resultó menos directivo y dominante, propiciando un clima de mayor libertad y apertura para el entrevistado. Así, lo dicho en el grupo se asumió como punto de inserción de lo que se produce y cambia socialmente.

Las sesiones se llevaron a cabo en el propio centro de trabajo, ya que es un lugar propicio, de fácil acceso y alejado de distractores, buscando, de todos modos, que fuera neutral. Cada sesión tuvo una duración aproximada de 90 minutos para evitar el cansancio discursivo. Para su ejecución, se diseñó un guion de preguntas temáticas con la finalidad de que las respuestas despertaran ideas entre los participantes y que generaran nuevas opiniones sobre el tema; se trató de obtener respuestas a cuestionamientos concretos, facilitando el intercambio de opiniones y puntos de vista relevantes para los objetivos de la investigación.

El procedimiento para la realización de la investigación consistió en intercalar las entrevistas con las observaciones y en dejar para el final las sesiones de los grupos de discusión, una vez que se lograba hacer el contacto con los sujetos y estos aceptaban colaborar. Al realizar la entrevista, se consensaba la posibilidad de la observación y la invitación al grupo de discusión, aunque, si bien todos aceptaron, no fue posible lograr el $100 \%$ de participación de los formadores convidados.

\section{Resultados}

El enfoque etnográfico de la investigación implicó iniciar el trabajo de campo con cuatro categorías etic: lo personal, la formación, lo laboral y lo profesional para recabar la información visual a partir de las observaciones, así como la verbal a través de las entrevistas y los grupos de discusión. Posteriormente, en el análisis, se empleó la triangulación metodológica y de informantes para garantizar su confiabilidad y confrontar la información desde distintas fuentes, lo que permitió una mejor interpretación de los datos. De allí surgieron cinco categorías emic, correspondientes a: las metas, la resiliencia, los aspectos distintivos, el deber ser y las áreas de oportunidad como se muestra en el mapa sobre la construcción de la identidad profesional.

Los aspectos personales de los formadores ofrecen un panorama de las fortalezas que poseen como individuos y son un elemento fundamental en la plataforma para ser un buen formador de docentes. Por ejemplo, en las entrevistas, expresaron: "Me siento realizado", "Me siento bastante satisfecho" y "Me siento feliz y plena". 
El bastión de la familia, sus creencias en los valores de la humanidad y en personajes emblemáticos, aunados a una vida plena y satisfactoria conforman un baluarte que complementa la formación y la experiencia de un formador para el ejercicio óptimo de sus funciones. En este sentido, los formadores expresaron frases como: "Mis hijas, mi familia y que la felicidad está en ti mismo", "Una persona muy vinculada a la familia"; además se declararon admiradores de personalidades de muy diverso orden como: Antón Makarenko, Gabino Barreda, Juan Manuel Serrat, Silvio Rodríguez, Alberto Cortés, Jorge Luis Borges, Martin Luther King y Octavio Paz.

En relación con los valores, los formadores expresan de diferentes formas un actuar ético. Esto último se expresó en frases como: “Que debemos de actuar bien, con ética", o en declaraciones como: "Yo siempre digo lo que pienso y hago lo que digo, la congruencia". Tales expresiones ponen de manifiesto su sentir y -de alguna manera- su hacer en la vida personal. Valores que son relevantes por sí mismos, y que, encarnados en quienes tienen la responsabilidad de formar docentes, se convierten en grandes pilares al respecto. Como afirma Bolívar (2007):

La identidad es un constructo conformado, a la vez, por factores racionales (cognitivos) y no racionales (emotivos y afectivos) donde los valores personales y profesionales están en el núcleo que, a su vez, se expresa en la motivación, actitud y compromiso con que el profesorado afronta su trabajo. (p.14)
La cordialidad, el asumir retos, el ser reflexivos y críticos pero propositivos, así como la adaptación a las circunstancias son actitudes que caracterizan a los formadores de docentes de la UPN en Tehuacán. Sin embargo, se debe reconocer que ciertas actitudes no contribuyen a la formación adecuada de los sujetos y, por el contrario, son obstáculos para una formación integral. Al respecto, los alumnos expresaron en los grupos de discusión: "Ahora por decirle esto, ya estoy reprobada" y "En este momento yo he tenido problemas, me han tronado por andar defendiendo a mis compañeros". Otra actitud observada y criticable fue el hecho de que en un examen profesional: "La Presidenta de los sinodales antes de iniciar el examen pidió a los presentes que desocuparan el salón por un momento mientras se ponían de acuerdo".

Las metas personales, familiares y profesionales que se proponen los formadores expresan la intención de ser mejores como personas, en su familia y como profesionales de la educación. Dicen en concreto: "Debo entrar a una edad adulta dignamente, que pueda estar plena, sana emocional y físicamente" o "Que pueda tener buena relación con mis hijas, con mi esposo". Lo que también se percibe cuando hablan de estudiar: "Continuar un estudio, el doctorado en educación o una especialidad", "Ser una académica reconocida" y "Lograr una productividad intelectual". Sobre este último tópico habría que destacar de manera contundente que los requerimientos profesionales apuntan a que los formadores buscan cursar posgrados con la in- 
tención de ser más productivos intelectualmente y obtener mayores reconocimientos, tanto sociales como económicos. Como plantea Giménez (1997), la identidad "tiene un carácter intersubjetivo y relacional. Es la autopercepción de un sujeto en relación con los otros; a lo que corresponde, a su vez, el reconocimiento y la 'aprobación' de los otros sujetos" (p.12).

La resiliencia mostrada por los formadores (entendida como capacidad de adaptarse y superar la adversidad) les permite librar los obstáculos y sobresalir ante los retos difíciles que las circunstancias les han puesto en su trayectoria tanto personal como laboral. Los ejemplos siguientes muestran las barreras que debieron superar: "De hecho era una práctica discriminatoria, cuando nosotros arribamos a la ciudad de Puebla"; "Se terminó el contrato y me dieron las gracias"; "De mi familia nadie estudió nada y yo sí tenía esa mentalidad, yo vengo de una familia muy humilde"; y "Fue difícil porque no tuve la fortuna de tener papá”. A la postre, estas situaciones les han resultado provechosas en su propia formación, dado que después las utilizan de manera positiva para propiciar una mejor preparación de sus alumnos. Y es que este tipo de aspectos se suman a la conformación de la identidad profesional de los formadores porque "La identidad (...) es algo que se negocia constantemente a lo largo de la vida" (Cacho, 2004, p.80).

Por otra parte, los sujetos investigados valoran la importancia de las diferentes insti- tuciones de educación superior en las que se formaron. Por ejemplo, los docentes entrevistados señalaron: "Soy educadora de formación en Escuela Normal", "Posteriormente también estudié la Maestría en Ciencias de la Educación y la Maestría en Investigación Educativa" y "Mi formación de origen es la Psicología". De esta manera, queda claro que la formación es fundamental para definir la identidad profesional del docente, la cual "Se va constituyendo fundamentalmente a partir de tres procesos: a) la construcción histórica de la profesión, b) la formación inicial y continua de los profesores, y c) sus actividades docentes cotidianas" (Cacho, 2004, p.79).

Pero, además, los formadores tienen en gran estima el valor formativo de la educación informal. En este sentido, comentaron en los grupos de discusión: "Otra manera de formarse es participando en las academias donde se analizan los aspectos académicos de los programas como de los propios alumnos" y "La universidad me ha dado mucho en cuanto a congresos, eventos, esa posibilidad de participar". Eventos, espacios y experiencia que van construyendo la identidad, pues, como destaca Dubar (2000): "Diversos procesos de socialización que, conjuntamente, construyen los individuos y definen las instituciones" (p.109). También Bolívar et al. (2005) se expresan en términos similares: "Las identidades se construyen, dentro de un proceso de socialización, en espacios sociales de interacción, mediante identificaciones y atribuciones" (p.3). 
La formación es una parte esencial del formador, el don de la enseñanza y el aprendizaje se reciben como condiciones de identidad: "Es un hecho práctico que permite establecer una ubicación reconocida con respecto a los aspectos importantes que definen el don del saber pedagógico y de la pedagogía" (Fayad, 2009, pp.85-86). El mismo Fayad (2008) reafirma: "Saber un saber y saberlo enseñar fundamenta la relación con el saber particular de las disciplinas y facultades" (p.94).

Al referirse a los profesores de las universidades y su papel en la formación, Sánchez (2008) cita uno de los postulados de Kant: "Salir de la minoría de edad. Tomar una decisión y tener el valor para servirse de sí con independencia. Servirse de su propio entendimiento" (p.131), pues, se trata de someter nuestras ideas al examen crítico de otros.

Ser mayor de edad no solo impone el uso de la razón (...). Implica la libertad (...). Lo cual nos propone dos exigencias: Una, hablar en el espacio público y dos, tener en cuenta el referente cultural, el acumulado de pensamiento. (p.132)

Las universidades pedagógicas deben formar docentes idóneos, es decir, con valores, reflexivos, participativos, creativos, comunicativos y humanistas. "En consecuencia, se requieren docentes sintonizados con las ideas anteriores, por lo que es necesario orientar a los futuros formadores en el mismo sentido en que han de actuar" (Ruedas, 2015).
El aspecto laboral de los formadores implica hablar de su contrato de trabajo en la UPN, que contempla diferentes vías de ingreso. Por ejemplo, ellos comentaron en las entrevistas: "Yo soy exalumno de esta institución y cuando terminé mis estudios aún sin titularme, me ofrecieron trabajar como asesor y acepté"; "Yo era el que cubría ese perfil, me invitaron y accedí" o "Hice examen de oposición y gané la plaza".

Aparte de este tipo de oportunidades, la experiencia docente también cuenta, es decir, la que se adquiere en otros ámbitos académicos asimismo es considerada, ya que de lo contrario solo trabajarían académicos de origen normalista. Al respecto, comentaron: "La mayoría o más bien todos ya han tenido experiencias en otros niveles educativos" y "No importa lo que haya estudiado, pues el profesor se hace en la práctica".

Esta riqueza de perfiles profesionales se ha conformado a través de más de 30 años, una identidad profesional propia de la UPN que, si bien comparte aspectos con otras instituciones y niveles educativos, también adquiere rasgos particulares que la identifican y diferencian. Como enfatiza Bolívar et al. (2005): "La identidad profesional se configura como el espacio común compartido entre el individuo, su entorno profesional y social y la institución donde trabaja" (p.4).

En el aspecto profesional de los formadores, las tareas de asesoría de tesis así como de tutoría 
de los alumnos son dos funciones que se desarrollan de manera directa con los alumnos y los formadores están conscientes de que destinan parte importante de su tiempo a esta actividad. Aun así, no perciben un reconocimiento institucional por esta labor. Los formadores opinaron en concreto: "Atendemos a los alumnos que egresan, asesorándolos para que se titulen”. También: "Realizamos tutorías con los alumnos de manera individual y en grupos para ayudarlos en los aspectos académicos y a veces hasta psicológicos o personales". En las observaciones llevadas a cabo durante las reuniones de academia los formadores hicieron apreciaciones como: "Respecto de las tutorías en los diferentes campos de formación con los alumnos del sexto semestre".

Pero, por distintas circunstancias, los formadores reconocen que hacen poca investigación: "No hago investigación porque requiere otros esfuerzos y por lo menos en este momento no creo poder desarrollarlos" y "Generalmente no hacemos investigación por falta de tiempo". Durante un coloquio se detectó que "Los académicos casi no participaron como ponentes, ni tampoco estuvo la mayoría en el evento". Y es que la investigación no se asume como una función sustantiva de la universidad, como plantea Sánchez (1995): "No hay investigadores por decreto, ni por nombramiento" (p.10).

En el modo de ver de los formadores, la función esencial de la universidad es la docencia, que se desarrolla en las aulas de la institución, lo que es una manifestación tácita de su formación y sus convencimientos, concretizada en los hechos tangibles que realizan en las horas clase con los alumnos. Algunos profesores aceptan la asunción de un enfoque tradicionalista. Por ejemplo, alguno expresó: "Tengo rasgos de profesor tradicional, me gusta hablar". Y en los grupos de discusión, los alumnos señalaron que: "Hay algunos profesores un poco autoritarios, pues quieren escuchar participaciones solo de lo que ellos piensan". En una clase se observó, además, que un alumno: "Solicita autorización para hacer la exposición en PowerPoint a lo cual el asesor le dice que no, que lo debe hacer en papel bond para que le vaya haciendo anotaciones".

Sin embargo, también hay elementos que muestran otros enfoques, como el constructivista: "Mi forma de trabajar es tipo seminario" y "Solicito que elaboren un mapa conceptual, una redacción, un ensayo". Se observó en las clases que el docente: "Indica que hagan un esquema de la lectura conforme vayan discutiendo con sus compañeros". También se observó cómo, de manera colegiada en un día completo de trabajo, se organiza institucionalmente con todos los alumnos: "La realización del taller integrador de la Licenciatura en Educación Indígena”.

Estos hechos van dejando huella en la formación de los nuevos docentes, quienes los reproducirán de alguna manera en su vida profesional próxima, como apunta Bolívar et al. (2005): “La identidad profesional suele ser resultado de un largo proceso por construir un modo propio de sentirse profesor, al tiempo que dan sentido a su ejercicio cotidiano" (p.14). 
Todos los formadores de docentes de la UPN en Tehuacán expresaron tener y sentir una vocación por la formación. Están orgullosos y satisfechos, y se sienten identificados con la institución: "La UPN es el gusto de estar aquí, en este espacio. Ese gusto de estar en una tarea que es esencial, de trabajar con gente que va a tener injerencia en cuestiones educativas". También como orgullo: "Creo que puedo decir orgullosamente soy un profesor" y "Fui adquiriendo ese deseo, ese gusto, ese interés porque además me representa una satisfacción".

Giménez (2005) considera que las identidades colectivas de los profesionales "Están constituidas por individuos vinculados entre sí por un sentimiento común de pertenencia" (p.29). Mercado (2004) coincide en ello cuando afirma que "La identidad profesional es aquella que (...) resulta de la conciencia de pertenencia a un grupo particular que se define en principio por oposición y diferencia con los otros" (p.103). Y Dubar (2002) concluye que "La identidad es la pertenencia común" (p.11).

En los aspectos distintivos, el reconocimiento social con que cuentan los formadores es el resultado del compromiso adquirido por ellos mismos al trascender socialmente por sus actividades en la universidad. He aquí algunas expresiones pertinentes: "Yo tengo claro que nosotros, los docentes universitarios, tenemos un compromiso con la sociedad"; "Me reconocen como una persona comprometida en lo que hago"; y "Cada uno de nosotros somos valorados por nuestros compañeros, por los alumnos y hasta de la sociedad de Tehuacán”.

Otro aspecto que los distingue es su sólida formación, los alumnos comentaron en el grupo de discusión: "Hay maestros que conocen su materia, que sí saben de lo que están hablando, hacen reflexión y análisis, de ellos son de los que yo considero que me han dejado una enseñanza".

De este modo, el trabajo académico realizado de manera profesional ha derivado en una buena imagen y en el reconocimiento de los méritos. Por ejemplo, los formadores comentaron: “Aquí todos tenemos buena imagen, pues, nos la hemos ganado" y "Todos los profesores independientemente de sus tiempos contratados realizan con profesionalismo su trabajo".

Tal condición, una vez definida y reconocida por los demás, se va constituyendo en la identidad profesional para cada individuo. En este sentido, Giménez (1997) afirma que la identidad profesional es "La capacidad de distinguirse y ser distinguido de otros grupos (...) de configurar y reconfigurar el pasado del grupo como una memoria colectiva compartida por sus miembros e incluso de reconocer ciertos atributos como propios y característicos" (p.18).

Los aspectos del deber ser, hacer y tener de los formadores se conjugan en una serie de elementos fundamentales que se manifiestan en 
la manera como se representan y actúan en su desempeño profesional. Sobre el particular, los formadores plantearon en las entrevistas que se debe lograr: "Esa coherencia entre lo que se dice y lo que se hace"; en tanto que en el hacer, hay que: "Impulsar la actualización docente de los asesores de la UPN en un espacio de diálogo y reflexión"; y en el tener, se debe configurar "Una cultura general sólida" y "Ser competente en el manejo de grupos". Mieles-Barrera et al. (2009) propone que estos aspectos del deber ser constituyen parte de la identidad profesional y los explica así:

La identidad profesional del docente puede abordarse desde el deber ser, es decir, de los retos, deberes y funciones que son necesarios asumir, en relación con las demandas del contexto, de la comunidad educativa y el proyecto de país que se plantea desde la Constitución Nacional. (pp.50-51)

Las áreas de oportunidad que se visualizan para los formadores requieren la organización y el compromiso institucional. Sobre el tema, los alumnos señalaron en los grupos de discusión: "No decimos nombres por ética profesional, pero hay asesores que llegan a agarrar el libro pero sin ninguna planeación”. Una situación concreta observada en un evento institucional consistió en que: "En el inicio de la conferencia existió desorganización, no se ponían de acuerdo quién tomaba la palabra, para el evento estaban dos presentadores improvisados". También señalaron los formadores que: "Gente de tiempo completo, no tiene compromiso, viven la vida relax, tranquilamente".

La puntualidad, los consensos, el trabajo en equipo y la productividad son aspectos interrelacionados e interdependientes, pues, cuando uno falla, interfiere de manera negativa en los demás; sin embrago, cuando logran articularse armónicamente hacen exitosa, eficiente y productiva a la institución. De esa forma se va constituyendo una identidad colectiva, a la cual pertenece la identidad profesional, como explica García (2003): "La identidad se construye y se reconstruye constantemente en el seno de los intercambios sociales" (p.256).

\section{Conclusiones}

Los aspectos más importantes del presente trabajo indican, en primer lugar, que un formador, a partir de su resiliencia ante la vida, sale avante en las situaciones que se le presentan en las actividades personales, familiares, laborales y profesionales. Tales experiencias, principalmente cuando representan una problemática mayor, se suelen convertir en una fortaleza para ayudar a formar a nuevos profesores.

Por otra parte, en las metas que se proponen los formadores, se reconoce a un ser humano común y corriente, que se siente preocupado por cuestiones básicas. También está presente la preocupación por la familia, ya que estando bien consigo mismo y con ella, podrán preocuparse por cuestiones profesionales, que de manera especial apuntan a los posgrados para 
ser productivos intelectualmente y reconocidos por los demás.

Además, la identidad profesional de los formadores está estrechamente vinculada con el ejercicio propiamente dicho de la docencia, de donde emanan sus mayores fortalezas y los aspectos que los definen. Se percibe una mezcla de enfoques que se desarrollan en las aulas de la institución como una manifestación tácita de sus formaciones y convencimientos, que se concretan en las horas de clase con los alumnos y que van dejando huella en la formación de los nuevos docentes, quienes las reproducirán de alguna manera en su vida profesional próxima.

Las tareas de asesoría de tesis, así como la de tutoría que se desarrollan de manera directa entre el formador y los alumnos. Por tanto, se convierten en espacios formativos por excelencia para ambos, que también van construyendo identidad profesional. Paradójicamente, sin embargo, la investigación como función sustantiva de la universidad está lejos de alcanzar los estándares mínimos para considerarse como tal.

También es de resaltar que sin importar si provienen de escuelas normales o de universidades, los formadores llegaron a la UPN, donde se les han brindado espacios de formación y han adquirido experiencia tanto en los salones de clases como en los diferentes espacios en los que se realizan eventos académicos y reuniones colegiadas; esto, aunado a su formación académica, forja aspectos que los definen como formadores de docentes.

Existen diferentes vías para tener un contrato laboral en una institución formadora de docentes como la UPN en Tehuacán, Puebla, desde la más ortodoxa, que consiste en presentar un examen de oposición, hasta la de aprovechar las oportunidades y las circunstancias.

La identidad que han construido los formadores frente a los retos y exigencias del contexto social y del Sistema Educativo Nacional pone al descubierto nuevas áreas que no se han cubierto de manera satisfactoria, lo que hace imperante la necesidad de atenderlas.

El ser, el hacer y el tener se conjugan en un deber que exige una serie de elementos fundamentales en la manera de ser y de actuar en el desempeño profesional del formador de docentes. Tales exigencias parecen simples, pero si no se manifiestan en evidencias palpables, tampoco serán reconocidas en su contexto inmediato y parecería, en tal caso, que no forman parte de ellos como identidad profesional.

Finalmente, los formadores se reconocen como tales, y sus compañeros y alumnos igualmente observan en ellos aspectos distintivos que los delimitan como formadores de docentes. Así, la identidad profesional no sólo es reconocida de forma personal, sino también colectiva. Con otras palabras se requiere del yo y del nosotros para ser al mismo tiempo identidad y alteridad. 


\section{Referencias}

Avalos, B. Cavada, P. Pardo, M. y Sotomayor, C. (2010). La Profesión Docente: Temas y Discusiones en la Literatura Internacional; Estudios Pedagógicos, 36(1), 35-263.

Bolívar, A., Fernández, M. y Molina, E. (2005). Investigar la identidad profesional del profesorado: Una triangulación secuencial. Forum: Qualitative Social Research, 6(1).

Bolívar, A. (2007). La formación inicial del profesorado de secundaria y su identidad profesional. Estudios sobre Educación, 13(12), 13-30.

Cacho, M. (2004). Profesores, trayectoria e identidades. Revista Latinoamericana de Estudios Educativos, 35(3), 69-111.

Cámara de Diputados LXII Legislatura. (1 septiembre 2013). Ley General del Servicio Profesional Docente. Gaceta Parlamentaria, Año XVI, Núm. 3851-A. México.

Cattonar, B. (2001). Les identités professionnelles enseignantes. ébauche d'un cadre d'analyse. Cahiers de Recherche $d u$ Girsef, 10, 1-34.

Díaz de Rada, Á. (2011). El taller del etnógrafo. Materiales y herramientas de investigación en Etnografia. Madrid: UNED.

Dubar, C. (2000). La socialisation. Construction des identités sociales et professionnelles. París: Armand Colin.

Dubar, C. (2002). La crisis de las identidades: la interpretación de una mutación. Barcelona: Bellaterra.
Fayad, J. A. (2008). El desempeño profesoral universitario lo pensamos como consecuencia de la flexibilización curricular, Revista Educación y Humanismo, 10 (15), 86-99.

Fayad, J. A. (2009). Adquirir el don de la pedagogía, significa defender la profesión docente, Revista Educación y Humanismo, 11 (16), 84-98.

García, A. (2003). Identidad y cultura: efectos en la educación intercultural. Pedagogía Social. Revista interuniversitaria, Segunda época, (10), 253-264.

Gewerc, Adriana (2001). Identidad profesional y trayectoria en la universidad. Profesorado, revista de currículum y formación del profesorado, 5(2).

Giménez, G. (1997). Materiales para una teoría de las identidades sociales. Frontera Norte, 9(18), 9-28.

Giménez, G. (2005). Teoría y análisis de la cultura, México: Conaculta.

Hammersley, M. y Atkinson, P. (2009). Etnografia. Métodos de investigación. Madrid: Paidós.

Mercado, L. (2004). Imbricación entre identidad y representaciones profesionales. Voces y miradas del profesor de primaria pública. En J. M. Piña (Ed.), La subjetividad de los actores de la educación (97-117). México: CESU-UNAM.

Messina, G. (1999). Investigación en o investigación acerca de la formación docente: un estado del arte en los noventa. Revista Iberoamericana de Educación, (19), 145-207. 
Mieles-Barrera, M. D., Henríquez-Linero, I. Ruedas, M. J. (2015). Aportes axiológicos de

M. y Sánchez-Castellón, L. M. (2009). Identidad personal y profesional de los docentes de preescolar en el distrito de Santa Marta. Educación y Educadores, 12(1), 43-59.

Presidencia de la República (19 mayo, 1992). Acuerdo Nacional para la Modernización de la Educación Básica. México: Diario Oficial de la Federación.

Rebollo, M. Á. González, E. \& García, M. R. (2002). Identidades profesionales en educación desde una perspectiva de género, Revista Fuentes No. 3.

Reese, L. Kroesen K. y Gallimore R. (2010). Cualitativos y cuantitativos, no cualitativos vs. Cuantitativos. En R. Mejía y S. A. Sandoval (Eds.), Tras las vetas de la investigación cualitativa. (39-75). Jalisco: ITESO.

Reynaga, S. (2010). Perspectivas cualitativas de investigación en ámbito educativo. En R. Mejía y S. A. Sandoval (Eds.), Tras las vetas de la investigación cualitativa (123-154). Jalisco: ITESO. experiencias didácticas complejizantes en la formación de docentes, Educación y Humanismo, 18(30) 28-41.

Sánchez, N. A. (2008). El Maestro Universitario: Un Mayor de Edad, Revista Educación y Humanismo, (14), 129-135.

Sánchez, R. (1995). Enseñar a investigar. Una didáctica nueva de la investigación cientifica en ciencias sociales y humanas. México: CESU-UNAM.

SEP. (2011). Acuerdo Número 592 por el que se establece la articulación de la Educación Básica. México: Author.

Vaillant, D. (2002). Formación de formadores. Estado de la práctica. Santiago de Chile: PREAL.

Valdés, R., Fernández, O. y da Silva, J. P. (2005). Estado de arte sobre formación de profesores en América Latina: Significado, orígenes y fundamentos teórico-metodológicos, Revista Digital UMBRAL 2000, 17.

Velasco, H. M. y Díaz de Rada, Á. (2009). La lógica de la investigación etnográfica. Madrid: Trotta. 